\title{
A familial late-onset hereditary ataxia mimicking pontocerebellar hypoplasia caused by a novel TSEN54 mutation
}

\author{
YI QIAN $^{1,2 *}$, HAIYONG WANG ${ }^{3 *}$, TAO JIN $^{4}$, YIQING WANG ${ }^{5}$, \\ LIZHENG FANG $^{2}$, YUHAO CHEN ${ }^{2}$ and LIYING CHEN ${ }^{2}$

\begin{abstract}
${ }^{1}$ School of Public Health, Fudan University, Shanghai 200032; ${ }^{2}$ Department of Family Medicine, Sir Run Run Shaw Hospital, School of Medicine, Zhejiang University, Hangzhou, Zhejiang 310016; ${ }^{3}$ Department of Oncology,

The First Affiliated Hospital, School of Medicine, Zhejiang University, Hangzhou, Zhejiang 310003;

${ }^{4}$ Department of Cardiothoracic Surgery, Zhejiang Provincial People's Hospital, Hangzhou, Zhejiang 310014;

${ }^{5}$ Department of Clinical Medicine, School of Medicine, Zhejiang University, Hangzhou, Zhejiang 310012, P.R. China
\end{abstract}

Received November 3, 2013; Accepted May 13, 2014

DOI: $10.3892 / \mathrm{mmr} .2014 .2342$

\begin{abstract}
Pontocerebellar hypoplasia $(\mathrm{PCH})$ comprises a rare group of neurodegenerative disorders with variable symptoms of cerebellar ataxia. Several gene mutations have been reported to be associated with different presentations of $\mathrm{PCH}$. In the present study, an extended familial case of late-onset hereditary ataxia mimicking $\mathrm{PCH}$ in respect of clinical manifestation, neuroradiological findings and genetic analysis is described. By means of direct sequencing, a novel heterozygous mutation was found in the TSEN54 gene by c.254A > T(+) (p.E85V), which may be a new subtype of hereditary ataxia. However, this subtype was shown to exhibit late onset, differing from $\mathrm{PCH}$ with prenatal onset and predominantly affecting the growth of neurons.
\end{abstract}

\section{Introduction}

Hereditary ataxia comprises a clinically and genetically heterogeneous group of neurodegenerative disorders (1). Clinical features include progressive limb and gait ataxia, loss of coordination and disturbance of speech and occulomotor control. Lesions are common in the spinal cord, cerebellum and brain stem; thus, the pathology is also referred to as spinocerebellar ataxia (SCA). In 1863, Friedreich (2) made the first report of a case of SCA that occurred in an adolescent, and since then it has been reported that several gene mutations

Correspondence to: Dr Liying Chen, Department of Family Medicine, Sir Run Run Shaw Hospital, School of Medicine, Zhejiang University, 3 Qingchundong Road, Hangzhou, Zhejiang 310016, P.R. China

E-mail: chenliying0609@gmail.com

${ }^{*}$ Contributed equally

Key words: pontocerebellar hypoplasia, TSEN54 mutation, hereditary ataxia are associated with various subtypes of hereditary ataxia. In this study, a familial case of late-onset hereditary ataxia was identified, mimicking pontocerebellar hypoplasia $(\mathrm{PCH})$ in its clinical, neuroradiological and genetic aspects, but differing in its age of onset. At present, seven different subtypes of PHC have been identified, which are commonly characterized by hypoplasia and variable atrophy of cerebellum and pons, progressive microcephaly and variable cerebral involvement. Mutations in three tRNA splicing endonuclease subunit genes (TSEN54, TSEN2 and TSEN34) were demonstrated to be responsible for $\mathrm{PCH} 2, \mathrm{PCH} 4$ and $\mathrm{PCH} 5$. Mutations in the mitochondrial tRNA arginyl synthetase gene (RARS2) are associated with PCH6. TSEN54, RARS2 and the vaccinia related kinase 1 (VRK1) are mutated in a minority of PCH1 cases. PHC7, the latest subtype, has not yet been clarified in detail $(3,4)$.

\section{Case report}

The proband was a 36-year-old female referred to the Department of Family Medicine of the Sir Run Run Shaw hospital due to a consistently unsteady gait for 6 years. The patient initially presented with a mildly imbalanced gait and incoordination at 30 years of age. The symptoms progressed with unsteady walking and a tendency to fall, accompanied by coughing upon drinking and slurred speech. No symptoms of headache, vomiting or dysphagia were reported.

A family history investigation revealed a four-generation family history with seven affected family members, who presented with similar symptoms of cerebellar ataxia, including unsteady gait, coughing upon drinking and slurred speech, in their mid-thirties and succumbed due to complications of $\mathrm{PCH}$ during their fifties or sixties (Fig. 1). No aggravation of the symptoms was found from one generation to the next. The genetic inheritance pattern was consistent with an autosomal dominant model.

Physical examination showed a normal appearance and stature; however, the neurological examination revealed a series of positive indicators of cerebellar ataxia, including a wide-based gait, difficulty walking in a straight line, 


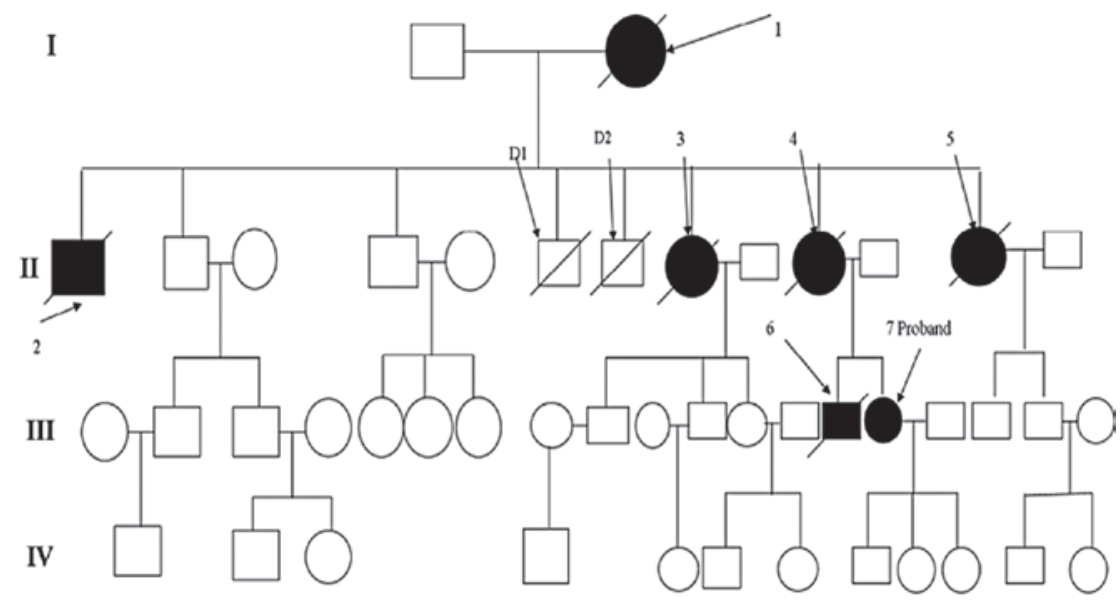

Figure 1. Pedigree of a Chinese family with pontocerebellar hypoplasia. Patient 1, onset at 30, diet at 50; patient 2, onset at 28, died at 60; patient 3, onset at 40 , died at 70; patient 4, onset at 30, died at 50; patient 5, onset at 30, died at 65; patient 6, onset at 27, died at 63; patient 7 (the proband), onset at 36 . Filled squares and circles denote affected males and females, respectively, indicated in the figure by arrows 1-7. Normal individuals are shown as empty symbols. The proband is shown by the indicated arrow. D1 died of cardiac problem in his fifties, D2 died of drowning in his twenties.

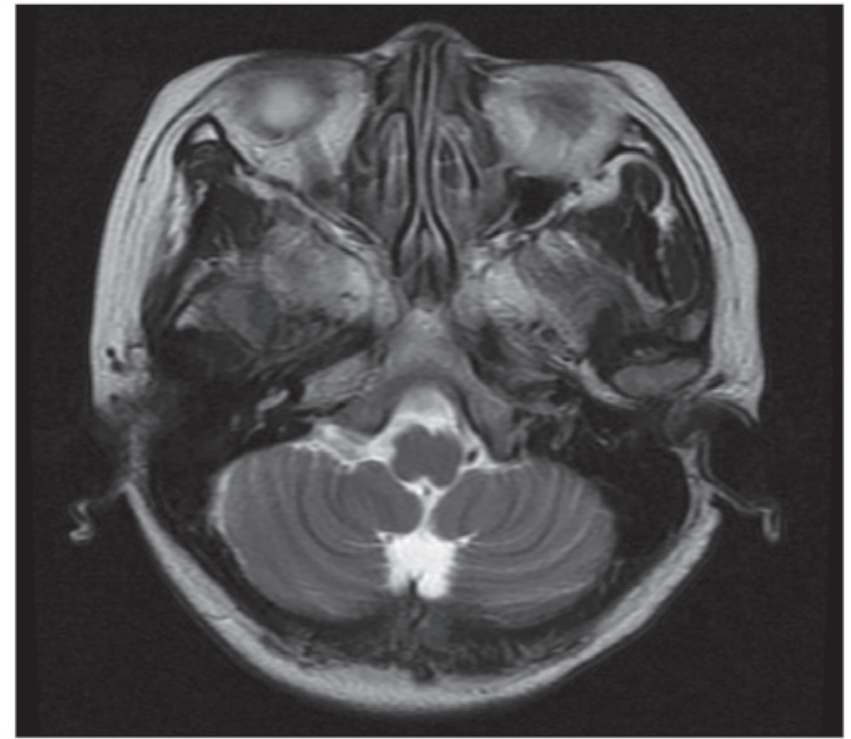

Figure 2. Brain magnetic resonance imaging scan of the proband, showing mild atrophy of the cerebellar hemisphere with visibly deepened sulci.

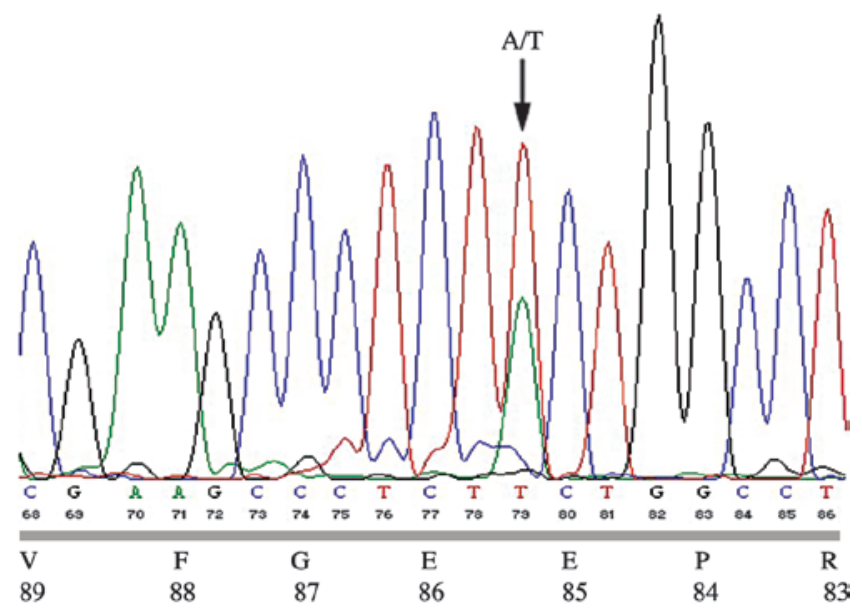

Figure 3. Sequencing chromatogram of TSEN54 gene. A heterozygous A254T transition was detected in the affected patients. V, valine; F, phenylalanine; G, glycine; E, glutamic acid; P, proline; R, arginine. hyporeflexia of the left pharynx reflex, enhanced muscular tension and tendon reflex of both lower extremities and positive results for the finger-to-nose and heal-knee-tibia tests, as well as for the Romberg and Babinski signs (tests of proprioception and pyramidal tract, respectively).

Initial laboratory investigations were normal, although magnetic resonance imaging (MRI) of the brain identified mild atrophy of the cerebellar hemisphere with visibly deepened sulci (Fig. 2). From these data, a primary diagnosis of hereditary cerebellar ataxia was considered.

A detailed gene analysis of the patient and other family members was conducted to determine further diagnosis and treatment. Prior to this, ethics approval was gained from the Ethics Committee of Zhejiang University (Hangzhou, China) and informed consent was obtained from the patient and all family members. Genomic DNA was extracted from nucleated blood cells of the affected and unaffected individuals of a four-generation Chinese family with $\mathrm{PCH}$. Candidate genes were then selected for sequencing based upon their reported involvement in PCH development. For mutation analysis, the coding region of TSEN54 was sequenced with DNA from one patient and other unaffected alive family members. Other susceptible sites, including two TSEN2 sites (c.926 A > G and c.960+1delGTAAG) and four mitochondrial arginyl-tRNA synthetase (RARS2) sites (c.35 A > G, c.110+5 A > G, c.1024 A > G and c.1072 C > T), were sequenced only in the affected patient. The primers were designed according to previous studies $(5,6)$. Amplification was performed using a Bori thermal cycler (Bori Technology Co., Ltd., Hangzhou, China) and polymerase chain reaction (PCR) products were then separated by polyacrylamide gel electrophoresis and directly sequenced using an $\mathrm{ABI} B \mathrm{BigDye}^{\circledR}$ Terminator Cycle Sequencing kit (Applied Biosystems, Austin, TX, USA) according to manufacturer's instructions and run on an ABI 3100 sequencer. Sequencing results were analyzed using the DNASTAR ${ }^{\circledR}$ software program package (DNASTAR, Inc., Madison, WI, USA).

A novel c.254A $>\mathrm{T}$ heterozygous mutation in the coding region of TSEN54 was found by direct sequencing in the 
affected family members. No other mutations were detected in any other exon of TSEN54, TSEN2, RARS2 or vVRK1 in the affected patient, and no mutations were found in any unaffected family members. The mutation resulted in an amino acid substitution from glutamic acid to valine at amino acid 85 (Fig. 3). For the differential diagnosis of SCAs, another hereditary ataxia disorder prevalent in the Chinese population, a CAG repeat mutation in SCA1, 2, 3, 6, 7 and 17 was screened for by PCR and DNA sequencing techniques. A CAG repeat was not found in any of the SCA genes in the affected patients.

\section{Discussion}

PCH comprises a group of neurodegenerative disorders characterized by a small cerebellum and brainstem and progressive microcephaly. The seven currently described PCH subtypes are distinguished by additional characteristics. Two subtypes, $\mathrm{PCH} 2$ and $\mathrm{PCH} 4$, are the most common, with dyskinesia and dystonia observed in $\mathrm{PCH} 2$ (7) and more severe symptoms and prenatal onset in $\mathrm{PCH} 4$. The most predominant clinical manifestation is cerebellar ataxia. Autonomic nervous-system disturbance and extra-pyramidal symptoms are also frequently observed in patients with PCH. Several genes have been shown to contribute to the manifestation and symptoms of $\mathrm{PCH}$, with all genes currently identified being involved in tRNA processing: $\mathrm{PCH} 2 \mathrm{~B}$ is caused by a TSEN2 mutation, $\mathrm{PCH} 2 \mathrm{C}$ is caused by TSEN34 mutations, PCH6 is caused by mutations in RARS2 (5) and mutations in TSEN54, the gene encoding noncatalytic subunits of the tRNA-splicing endonuclease complex, have been detected in patients exhibiting symptoms characteristic of $\mathrm{PCH} 2$ and $\mathrm{PCH} 4$ (6). A total of $>20$ mutations have been identified within the TSEN54 gene in various subtypes of $\mathrm{PCH}$, with A307S being the most commonly occurring mutation in families with inherited PCH (3).

The radiological features of $\mathrm{PCH}$ are hypoplasia and variable atrophy of the cerebellum and the pons. By MRI, the typical characteristic of $\mathrm{PCH} 2$ is a 'dragonfly-like' cerebellar pattern (4), while patients with $\mathrm{PCH} 4$ exhibit C-shaped inferior olives (8). The case presented in this study showed a mild atrophy of the cerebellar hemisphere with visibly deepened sulci; these radiological symptoms may correspond with the milder symptoms and late onset. It is difficult to differentiate $\mathrm{PCH}$ from other hereditary ataxia diseases from their clinical manifestations. SCA is a relatively common neurological degenerative disease with hereditary disparity. Abnormal CAG repeats found in the coding regions of SCA1, 2, 3, 6, 7 and 17 and DRPLA have been cloned (9). No abnormal CAG repeats were found in any of the affected patients in the present case.

VRK1 has been reported to be associated with a mild presentation of PCH1 (10), and mutations in the transfer RNA splicing endonuclease subunit genes TSEN54, TSEN2 and TSEN34 have been associated with $\mathrm{PCH} 2$ and 4 . In addition, mutations in RARS2 have been identified in patients with PCH6 (11).

TSEN54 is highly expressed in neurons of the pons, cerebellar dentate and olivary nuclei during the second trimester of pregnancy (12). Budde et al (11) identified homozygosity for an A307S mutation in the TSEN54 gene in 42/47 individuals with $\mathrm{PCH} 2$. In the presented case, a novel heterozygous missense c.254 $\mathrm{A}>\mathrm{T}$ mutation in the coding region of TSEN54 was identified. The consequences of this mutation on protein conformation and function and whether this results in hereditary ataxia mimicking $\mathrm{PCH}$ require further investigation. It is therefore important to perform a mutation analysis of known $\mathrm{PCH}$-causing genes in patients presenting with symptoms similar to $\mathrm{PCH}$.

\section{Acknowledgements}

This study was supported by a grant from the Health Bureau of Zhejiang Province, China (no. 2012KYA108).

\section{References}

1. Sumathipala DS, Abeysekera GS, Jayasekara RW, Tallaksen CM and Dissanayake VH: Autosomal dominant hereditary ataxia in Sri Lanka. BMC Neurol 13: 39, 2013.

2. Friedreich N: On degenerative atrophy of the spinal dorsal columns. Arch Pathol Anat Physiol Klin Med 26: 391-419, 1863 (In German).

3. Namavar Y, Barth PG, Poll-The BT and Baas F: Classification, diagnosis and potential mechanisms in pontocerebellar hypoplasia. Orphanet J Rare Dis 6: 50, 2011.

4. Namavar Y, Barth PG, Kasher PR, et al: Clinical, neuroradiological and genetic findings in pontocerebellar hypoplasia. Brain 134: 143-156, 2011.

5. Barth PG, Blennow G, Lenard HG, et al: The syndrome of autosomal recessive pontocerebellar hypoplasia, microcephaly, and extrapyramidal dyskinesia (pontocerebellar hypoplasia type 2): compiled data from 10 pedigrees. Neurology 45: 311-317, 1995.

6. Maricich SM, Aqeeb KA, Moayedi Y, et al: Pontocerebellar hypoplasia: review of classification and genetics, and exclusion of several genes known to be important for cerebellar development. J Child Neurol 26: 288-294, 2011.

7. Simonati A, Cassandrini D, Bazan D and Santorelli FM: TSEN54 mutation in a child with pontocerebellar hypoplasia type 1 . Acta Neuropathol 121: 671-673, 2011.

8. Barth PG, Aronica E, de Vries L, et al: Pontocerebellar hypoplasia type 2: a neuropathological update. Acta Neuropathol 114: 373-386, 2007.

9. Schöls L, Bauer P, Schmidt T, Schulte T and Riess O: Autosomal dominant cerebellar ataxias: clinical features, genetics, and pathogenesis. Lancet Neurol 3: 291-304, 2004.

10. Renbaum P, Kellerman E, Jaron R, et al: Spinal muscular atrophy with pontocerebellar hypoplasia is caused by a mutation in the VRK1 gene. Am J Hum Genet 85: 281-289, 2009.

11. Cassandrini D, Cilio MR, Bianchi M, et al: Pontocerebellar hypoplasia type 6 caused by mutations in RARS2: definition of the clinical spectrum and molecular findings in five patients. J Inherit Metab Dis 36: 43-53, 2013.

12. Budde BS, Namavar Y, Barth PG, et al: tRNA splicing endonuclease mutations cause pontocerebellar hypoplasia. Nat Genet 40: 1113-1118, 2008 